\title{
Field Quantization on Noncommutative Phase Space
}

\author{
Haihua $\mathrm{Hu}$ \\ Information and Electrical Engineering Department \\ Institute \\ Zhejiang University City College \\ Hangzhou 310015, PR China
}

\author{
Xiaohua Cao \\ Information and Electrical Engineering Department \\ Institute \\ Zhejiang University City College \\ Hangzhou 310015, PR China
}

\begin{abstract}
We introduce the foundational aspects of noncommutative quantum mechanics (NCQM). In order to develop the NCQM formulation we need to specify the phase space. We consider both space-space noncommutativity (space-time noncommutativity is not considered) and momentum-momentum noncommutativity as for the phase space. Then we study the second quantization of quantum field on noncommutative (NC) phase space and the energy of electromagnetic field is given when NC effect acts on.
\end{abstract}

Keywords-non-commutative; Field Quantization; annihilation-creation operators; quantum mechanics; Bose-Einstein statistics

\section{INTRODUCTION}

When one studies the low energy effective theory of D-brane with B field background, the very tiny string scale or at very high energy situation, the effects of noncommutativity of space may appear. In literature the $\mathrm{NC}$ quantum mechanics and $\mathrm{NC}$ quantum field theory have been studied extensively, in literatures many interesting topics of $\mathrm{NC}$ quantum theories have been extensively investigated, from the Aharonov-Bohm effect to the quantum Hall effect [1-7]. Recently quantum theory of NC fields has been proposed as a possible field theory generalization of $\mathrm{NC}$ quantum mechanics [8-11]. In this paper, we devote our mind to discuss the energy of electromagnetic field on $\mathrm{NC}$ phase space. We find appropriate to start our discussion based on the representation of the $\mathrm{NC}$ coordinates and $\mathrm{NC}$ momenta in terms of the commutative coordinates and momenta of usual quantum field. Let us denote the canonical coordinates and momenta operators

of quantum field harmonic oscillator $\left(k, \omega_{k}\right)$ in NC phase space as $\hat{Q}$ and $\hat{P}$ respectively, then the $\hat{Q}_{i}$ and $\hat{P}_{i}$ will have the following algebra relations, if both space-space and momentum-momentum non-commutativities are considered [12-14].

$$
\begin{aligned}
& \left\lfloor\hat{Q}_{i}, \hat{Q}_{j}\right\rfloor=i \theta \varepsilon_{i j},\left\lfloor\hat{Q}_{i}, \hat{P}_{j}\right\rfloor=i \hbar \delta_{i j}, \\
& {\left[\hat{P}_{i}, \hat{P}_{j}\right]=i \bar{\theta} \varepsilon_{i j}}
\end{aligned}
$$

where $\theta$ and $\bar{\theta}$ are totally very small matrix elements representing the $\mathrm{NC}$ property of the space and momentum in noncommutative phase space. On NC phase space the representations of $\hat{Q}$ and $\hat{P}$ in terms of $Q$ and $P$ were given as follows [15]

$$
\begin{aligned}
& \hat{Q}_{i}=\alpha Q_{i}-\frac{1}{2 \alpha \hbar} \theta \varepsilon_{i j} P_{j} \\
& \hat{P}_{i}=\alpha P_{i}-\frac{1}{2 \alpha \hbar} \bar{\theta} \varepsilon_{i j} Q_{j}
\end{aligned}
$$

Where $Q_{i}$ and $P_{j}$ are the canonical coordinates and momenta in commutative case, which have the following commutation relations:

$$
\begin{aligned}
& \left\lfloor Q_{i}, Q_{j}\right\rfloor=0,\left\lfloor Q_{i}, P_{j}\right\rfloor=i \hbar \delta_{i j}, \\
& {\left[P_{i}, P_{j}\right]=0}
\end{aligned}
$$

The $\alpha$ here is a scaling constant related to the noncommutativity of phase space and the three parameters $\theta, \bar{\theta}$,and $\alpha$ are related by [16]

$$
\bar{\theta}=4 \hbar^{2} \alpha^{2}\left(1-\alpha^{2}\right) / \theta
$$

According to NC canonical coordinates and momenta $\hat{Q}$ and $\hat{P}$, on NC phase space the Hamiltonian of a quantum electromagnetic harmonic oscillator $\left(k, \omega_{k}\right)$ can be given easily

$$
H_{k}=\frac{1}{2}\left(\hat{P}_{k}^{2}+\omega_{k}^{2} \hat{Q}_{k}^{2}=E_{k}\right.
$$

The three-dimensional magnetic vector $A$ can be expressed as Fourier expansion series 
$A(x, t)=\frac{1}{\sqrt{V}} \sum_{k} \frac{1}{\sqrt{2 \omega_{k}}} \sum_{\lambda=1}^{2} \varepsilon(k, \lambda)$

$\times\left[a_{k \lambda} \exp (-i k \cdot x)+a_{k \lambda}^{+} \exp (i k \cdot x)\right]$

Where $\omega_{k}=|k|, \quad \varepsilon(k, 1)$ and $\varepsilon(k, 2)$ are two orthogonal unit polarization vector, they are perpendicular to the wave vector $k$ of the plane wave.

The deformed light quantum annihilation-creation operators are defined by

$$
\begin{aligned}
& \hat{a}_{k \lambda}=\sqrt{\frac{\omega_{k}}{2 \hbar}} \hat{Q}_{k \lambda}+i \frac{\hat{P}_{k \lambda}}{\omega_{k}} \\
& \hat{a}_{k \lambda}^{+}=\sqrt{\frac{\omega_{k}}{2 \hbar}} \hat{Q}_{k \lambda}-i \frac{\hat{P}_{k \lambda}}{\omega_{k}} \\
& \lambda=1,2
\end{aligned}
$$

It is easy to check that,

$$
\begin{aligned}
& {\left[a_{k m}, a_{k n}^{+}\right]=\delta_{m n}+i \frac{\omega_{k}}{\hbar} \theta \varepsilon_{m n}} \\
& {\left[a_{k m}, a_{k n}\right]=\left[\stackrel{+}{a_{k m}, a_{k n}^{+}}\right]=\frac{i}{2 \hbar} \omega_{k}\left(\theta-\frac{1}{\omega_{k}^{2}} \bar{\theta}\right) \varepsilon_{m n}}
\end{aligned}
$$

In order to keep Bose-Einstein statistics in noncommutative case we need $\hat{a}_{k \lambda}$ and $\hat{a}_{k \lambda}^{+}$to be commuting, whence the consistency condition:

$$
\bar{\theta}=\omega_{k}^{2} \theta
$$

Under this condition, the above commutators become

$$
\left[a_{k \lambda}, a_{k \lambda}^{+}\right]=1
$$$$
\left[a_{k m}, a_{k n}\right]=\left[a_{k m}^{+}, a_{k n}^{+}\right]=0
$$

And then the annihilation-creation operators on $\mathrm{NC}$ phase space become

$$
\begin{aligned}
& a_{k m}=\alpha a_{k m}+\frac{i}{2 \alpha \hbar} \omega_{k} \theta \varepsilon_{m n} a_{k n} \\
& \stackrel{+}{a_{k m}}=\alpha a_{k m}^{+}-\frac{i}{2 \alpha \hbar} \omega_{k} \theta \varepsilon_{m n} a_{k n}^{+}
\end{aligned}
$$

Where $a_{k \lambda}$ and $a_{k \lambda}^{+}$is defined as the annihilation-creation operators on commutative case

$$
\begin{aligned}
& a_{k \lambda}=\sqrt{\frac{\omega_{k}}{2 \hbar}}\left(Q_{k \lambda}+i \frac{Q_{k \lambda}}{\omega_{k}}\right) \\
& a_{k \lambda}^{+}=\sqrt{\frac{\omega_{k}}{2 \hbar}}\left(Q_{k \lambda}-i \frac{Q_{k \lambda}}{\omega_{k}}\right)
\end{aligned}
$$

Up to now, we have given the representation of creation-annihilation operators of noncommutative field in terms of the ones in the usual commutative quantum field. Using these representations we can study the effects of non-commutativity in the usual space we are familiar with. Now we can discuss the Hamiltonian and angular momentum in term of annihilation and creation operators. It is easy to check that the Hamiltonian of quantum electromagnetic harmonic oscillator in equation (5) has the form

$$
\begin{gathered}
H_{k}=\sum_{\lambda=1}^{2}\left(a_{k \lambda}^{+} a_{k \lambda}+\frac{1}{2}\right) \hbar \omega_{k} \\
=\left[\left(\alpha a_{k 1}^{+}-\frac{i}{2 \hbar \alpha} \omega_{k} \theta a_{k 2}^{+}\right)\left(\alpha a_{k 1}+\frac{i}{2 \alpha \hbar} \omega_{k} \theta a_{k 2}\right)\right.
\end{gathered}
$$

$\left.+\left(\alpha a_{k 2}^{+}+\frac{i}{2 \hbar \alpha} \omega_{k} \theta a_{k 1}^{+}\right)\left(\alpha a_{k 2}-\frac{i}{2 \alpha \hbar} \omega_{k} \theta a_{k 1}\right)+1\right] \hbar \omega_{k}$

$=\left[\left(\alpha^{2}+\frac{\omega_{k}^{2} \theta^{2}}{4 \alpha^{2} \hbar^{2}}\right) a_{k 1}^{+} a_{k 1}+\left(\alpha^{2}+\frac{\omega_{k}^{2} \theta^{2}}{4 \alpha^{2} \hbar^{2}}\right) a_{k 2}^{+} a_{k 2}-\frac{i}{\hbar} \omega_{k} \theta\left(a_{k 2}^{+} a_{k 1}-a_{k 1}^{+} a_{k 2}\right)+1\right] \hbar \omega_{k}$

According to equation (6), we can obtain the third component of non-normal order photon spin operator $S$

$$
S_{3}=i\left(a_{k 2}^{+} a_{k 1}-a_{k 1}^{+} a_{k 2}\right)
$$

For the noncommutativity of quantum field, the energy of a quantum electromagnetic harmonic oscillator $\left(k, \omega_{k}\right)$ can be expressed as follow

$E_{k}=H_{k}=\sum_{\lambda=1}^{2}\left[\left(\alpha^{2}+\frac{\omega_{k}^{2} \theta^{2}}{4 \alpha^{2} \hbar^{2}}\right) n_{k \lambda}-\frac{\omega_{k} \theta}{2 \hbar} S_{3}+\frac{1}{2}\right] \hbar \omega_{k}$

From (4) and (9), we have

$\theta=\frac{2 \alpha \hbar}{\omega_{k}} \sqrt{1-\alpha^{2}}$

Then

$H_{k}=\sum_{\lambda=1}^{2}\left(n_{k \lambda}-\alpha \sqrt{1-\alpha^{2}} S_{3}+\frac{1}{2}\right) \hbar \omega_{k}$

We define the creation-annihilation operator of left and right polarized light $a_{k \lambda}$ and $a_{R}^{+}, a_{L}^{+}, a_{R}$ and $a_{L}$ as [17] 


$$
\begin{aligned}
& a_{k L}=\frac{1}{\sqrt{2}}\left(a_{k 1}+i a_{k 2}\right), a_{k R}=\frac{1}{\sqrt{2}}\left(a_{k 1}-i a_{k 2}\right) \\
& a_{k L}^{+}=\frac{1}{\sqrt{2}}\left(a_{k 1}^{+}-i a_{k 2}^{+}\right), a_{k R}^{+}=\frac{1}{\sqrt{2}}\left(a_{k 1}^{+}+i a_{k 2}^{+}\right)
\end{aligned}
$$

Obviously the third component of non-normal order photon spin operator $S$ has this form

$$
S_{3}=a_{R}^{+} a_{R}-a_{L}^{+} a_{L}
$$

It is easy to check that the Hamiltonian in equation (5) has the form

$$
H_{k}=\left(n_{k R}+\frac{1}{2}\right) \hbar \omega_{k R}+\left(n_{k L}+\frac{1}{2}\right) \hbar \omega_{k L}
$$

Where

$$
\omega_{k R}=\left(1-\alpha \sqrt{1-\alpha^{2}}\right) \omega_{k}, \omega_{k L}=\left(1+\alpha \sqrt{1-\alpha^{2}}\right) \omega_{k}
$$

Hence, the energy of whole electromagnetic field on NC phase space can be expressed as

$$
H=\sum_{k}\left[\left(n_{k R}+\frac{1}{2}\right) \hbar \omega_{k R}+\left(n_{k L}+\frac{1}{2}\right) \hbar \omega_{k L}\right]
$$

It is evident that the frequency shift is a sum of this paper. In fact, we should point out that the noncommutative effects always depend on the coordinates commutator matrix element $\theta$ which depends on the scaling constant $\alpha$. We introduce the basic relationship on NC phase space, which is based on the second quantization of quantum field, and deduce the corresponding $\mathrm{NC}$ effect. The significance of the second quantization is represented explicitly from our discussion. It should be pointed out that most of our results are not Lorentz invariant, because the time and the noncommutative spatial coordinates are assumed to be commutative in this paper. The method imported in this paper is also helpful to study many new quantum and optics effects when light propagates in new artificial electromagnetic media.

This work was supported partially Scientific Research Fund of Zhejiang Provincial Education Department of China (Y201223380)

\section{References}

[1] M. Chaichian, A. Demichev, P. Presnajder, M.M. Sheikh-Jabbari, A. Tureanu Phys. Lett. B527, 149 (2002) hep-th/0012175.

[2] M. Chaichian, A. Demichev, P. Presnajder, M.M. Sheikh-Jabbari, A. Tureanu Nucl. Phys. B611, 383 (2001) hep-th/0101209.

[3] S. S. Gubser, M. Rangamani, JHEP 0105: 041 (2001) hep-th/0012155.

[4] L. Susskind, hep-th/0101029.

[5] A. P. Polychronakos , JHEP 0104: 011 (2001) hep-th//0103013.

[6] S. Hellerman, M. V. Raamsdonk, JHEP 0110: 039 (2001) hep-th/0103179.

[7] H. Falomir, J. Gamboa, M. Loewe, F. Mendez, J.C. Rojas, Phy. Rev. D66, 045018 (2002) hep-th/0203260.

[8] J.M. Carmona, J.L. Cortes, J. Gamboa, F. Mendez, JHEP 0303 (2003) 058, [hep-th/0301248].

[9] V.P. Nair, A.P. Polychronakos, Phys.Lett.B505 (2001) 267, [hep-th/0011172].

[10] S. Bellucci, A. Nersessian, C. Sochichiu, Phys.Lett.B522 (2001) 345, [hep-th/0106138].

[11] C. Acatrinei, JHEP 0109 (2001) 007, [hep-th/0107078].

[12] V. P. Nair, A. P. Polychronakos, Phys. Lett. B505, 267 (2001) hep-th/0011172

[13] Jian-zu Zhang, Phys. Lett. B584, 204 (2004) hep-th/0405135

[14] M. R. Douglas, N. A. Nekrasov, Rev. Mod. Phys. 73, 977 (2001) hep-th/0106048.

[15] Jian-zu Zhang, Phys. Rev. Lett. 93, 043002 (2004) hep-ph/0405143.

[16] Kang Li, Jianhua Wang, Chiyi Chen, Mod. Phys. Lett. A Vol. 20, No. 28(2005) 2165-2174

[17] J.D.Bjorken, S.D.Drell, Relativistic Quantum Fields (1965) Chap.14 